\title{
BMJ Open Impact of non-pharmaceutical interventions for reducing transmission of COVID-19: a systematic review and meta-analysis protocol
}

Krishna Regmi (D) , ${ }^{1}$ Cho Mar Lwin ${ }^{2}$

To cite: Regmi K, Lwin CM. Impact of non-pharmaceutical interventions for reducing transmission of COVID-19: a systematic review and metaanalysis protocol. BMJ Open 2020;10:e041383. doi:10.1136/ bmjopen-2020-041383

- Prepublication history and supplemental material for this paper are available online. To view these files, please visit the journal online (http://dx.doi. org/10.1136/bmjopen-2020041383).

Received 06 June 2020 Revised 24 September 2020 Accepted 05 0ctober 2020

Check for updates

(C) Author(s) (or their employer(s)) 2020. Re-use permitted under CC BY-NC. No commercial re-use. See rights and permissions. Published by BMJ.

${ }^{1}$ Institute for Health Research, Faculty of Health and Social

Sciences, University of Bedfordshire, Luton, UK

2Department of Rheumatology, University of Medicine Mandalay, Mandalay, Myanmar

Correspondence to

Dr Krishna Regmi;

Krishna.r.regmi@gmail.com

\section{ABSTRACT}

Introduction Implementing non-pharmaceutical interventions (NPIs) protect the public from COVID-19. However, the impact of NPIs has been inconsistent and remains unclear. This study, therefore, aims to measure the impact of major NPIs (social distancing, social isolation and quarantine) on reducing COVID-19 transmission.

Methods and analysis We will conduct a systematic review and meta-analysis research of both randomised and non-randomised controlled trials. We will undertake a systematic search of: MEDLINE, Embase, Allied \& Complementary Medicine, COVID-19 Research, WHO database on COVID-19, ClinicalTrails. Gov for clinical trials on COVID-19, Cochrane Resources on Coronavirus (COVID-19), Oxford COVID-19 Evidence Service and Google Scholar for published and unpublished literatures on COVID-19 including preprint engines such as medRxiv, bioRxiv, Litcovid and SSRN for unpublished studies on COVID-19 and will be reported in accordance with Preferred Reporting Items for Systematic Reviews and Meta-Analyses. Outcomes of interest for impact analysis will include the reduction of COVID-19 transmission, avoiding crowds and restricting movement, isolating ill and psychological impacts. The Preferred Reporting Items for Systematic Review and Meta-Analysis Protocols checklist has been used for this protocol. For quality of included studies, we will use the Cochrane Collaboration's tool for assessing risk of bias for randomised controlled trials and the Newcastle-Ottawa Scale for observational studies. The Grading of Recommendations Assessment, Development and Evaluation approach will grade the certainty of the evidence for all outcome measures across studies. Random-effects model for meta-analysis will measure the effect size of NPIs or the strengths of relationships. For quantitative data, risk ratio or $\mathrm{OR}$, absolute risk difference (for dichotomous outcome data), or mean difference or standardised mean difference (for continuous data) and their $95 \%$ Cls will be calculated. Where statistical pooling is not possible, a narrative synthesis will be conducted for the included studies. To assess the heterogeneity of effects, $I^{2}$ together with the observed effects will be evaluated to provide the true effects in the analysis. Ethics and dissemination Formal ethical approval from an institutional review board or research ethics committee is not required as primary data will not be collected. The final results of this study will be published in an open-access peer-reviewed journal, and abstract will be
Strengths and limitations of this study

- To the best of our knowledge, this study is the first systematic review to measure the impact of non-pharmaceutical interventions (NPIs)-social distancing, isolation and quarantine - on reducing COVID-19 transmission.

- This study will offer the highest level of evidence to assist policymakers and researchers in synthesising a large and complex literature, drawing a broader framework.

- This protocol reduces the possibility of duplication, provides transparency to the methods and procedures used, minimises potential biases and allows peer review.

- This research is not externally funded, and therefore time and resource will be constrained.

- If included studies vary in sample size, quality and population, they may be open to bias, and the heterogeneity of data will preclude a meaningful metaanalysis to measure the effects of specific NPIs.

presented at suitable national/international conferences or workshops. We will also share important information with public health authorities as well as with the WHO. In addition, we may post the submitted manuscript under review to medRxiv, or other relevant preprint servers. Trial registration number CRD42020207338.

\section{INTRODUCTION}

COVID-19 (caused by SARS-CoV-2) emerged in Wuhan, China, in December 2019, and has been posing a global public health threat. On 11 March 2020, WHO declared the COVID-19 outbreak a pandemic. ${ }^{1}$ At the time of writing (24 September 2020), the WHO COVID-19 Situation Dashboard reports that this virus has already affected 216 countries, areas or territories with 31664104 confirmed cases of COVID-19 and 972221 deaths and a fatality rate of approximately over $3 \%(3.07 \%){ }^{2}$

Based on reported cases, approximately 1:10 reported infections were among healthcare professionals, for example, medical doctors 
and nurses. ${ }^{3}$ We have seen disproportionate numbers of black, Asian and minority ethnic (BAME) doctors and other healthcare professionals die from COVID-19. A study conducted by Cook et al reported that in the UK National Health Service, it is estimated that $21 \%$ of all staff are BAME, whereas $63 \%$ of healthcare professionals who died were BAME. A recent UK government review ${ }^{5}$ highlighted that the highest age-standardised diagnosis rates of COVID-19 per 100000 population were in people of BAME groups (486 female and 649 male) and the lowest were in white people (220 female and 224 male). Accounting for the effect of sex, age, deprivation and region, Bangladeshi people had about twice the risk of death compared with white British. Similarly, Chinese, Indian, Pakistani, other Asian, Caribbean and other black ethnicity had between $10 \%$ and $50 \%$ higher risk of death compared with white British. In fact, this is the opposite of observations in previous years, when all-cause mortality rates were lower in BAME. ${ }^{35}$

Similarly, the COVID-19 mortality rate in the USA for African-Americans was 2.4-2.7 times more than for white individuals. ${ }^{3}$ However, death rates are not consistent across these groups. Inequalities in COVID-19 mortalities are rife, which is most recently shown by Public Health England. ${ }^{6}$ Several factors were identified as risks for COVID-19, for example, ethnicity, age, sex, comorbidities (diabetes and renal conditions), occupation, socioeconomic status, and multifamily and multigenerational households. $^{6-8}$

Recent data from Johns Hopkins University reported that global COVID-19 deaths have surpassed $890000 .^{9}$ Imperial College London highlights that this outbreak could kill 40 million people this year without public health measures (eg, case finding, contact tracing and testing, and strict quarantine) ${ }^{7}$ Evidence suggests that the number of cases reported would possibly 'represent an underestimation of the true burden due to lack of surveillance and diagnostic capacity ${ }^{8}$ as well as pharmaceuticals to manage severe COVID-19. ${ }^{10}$

Several countries, including the UK, USA and other European Union countries are adopting social distancing (SD) measure as a form of non-pharmaceutical or physical interventions to control COVID-19 by slowing down transmission of the virus and preventing associated illness and death. ${ }^{11} \mathrm{SD}$ is the new buzzword with the outbreak of coronavirus and COVID-19. In the literature, the term SD can have different meanings; for example, some considered it as strategy, ${ }^{12}$ policy, ${ }^{13}$ an approach to flatten the curve, ${ }^{14}$ mitigation measure to increase physical distance or reduce frequency of congregation in socially dense community settings. ${ }^{15-17}$ Flaxman $e t a l^{10}$ defined SD as a measure to ban large gatherings and advise individuals not to socialise outside their households by closing borders, some public places, schools and universities; isolation/ quarantine, physical distancing and room separation to isolate symptomatic individuals and their contacts; and large-scale lockdowns of populations by staying at least $2 \mathrm{~m}$ apart aiming to minimise mixing of infectious susceptible patients. This definition of $\mathrm{SD}$, in fact, is very vague and includes interventions that are considered different to SD, for example, quarantine including school closure and case findings.

For clarity, in this systematic review (SR), the definition of SD (also called physical distancing) is considered as a set of non-pharmaceutical interventions (NPIs) intended to prevent spread of COVID-19 by maintaining physical distance between people and reducing the number of times people come into close contact. ${ }^{18} 19$ This review focuses only on COVID-19/SARS-CoV-2 and three major NPIs, namely SD, isolation and quarantine. Isolation of cases refers to the separation of ill persons with contagious diseases from non-infected persons, either hospitalised (moderate or severe cases) to provide care or in dedicated isolation facilities or at home (mild cases), ${ }^{20}$ and quarantine is the restriction of persons who are presumed to have been exposed to a contagious disease but are not ill, either because they did not become infected or because they are still in the incubation period. ${ }^{21}$ WHO recommends isolation, physical (social) distancing, contact tracing and quarantine of close contacts as the key measures to reduce COVID-19. ${ }^{22}$

A scoping search of MEDLINE was done on 9 September 2020 for publications entered by the end of August 2020 with the following terms: ("COVID-19" OR "SARS-CoV-2") AND ("systematic review" OR "literature search" OR "meta-analysis" OR "evidence synthesis") AND ("social distancing" OR "isolation" OR "quarantine")). It revealed some empirical research on COVID-19 from China, South Korea, the UK, the USA and other countries, but these are not systemically reviewed or synthesised well. Several rapid reviews and summaries have been covered on COVID-19 epidemiology, ${ }^{23}{ }^{24}$ the effectiveness of real-time PCR for diagnosis, ${ }^{25}$ effects of school closure, ${ }^{26}$ quarantine, ${ }^{27}{ }^{28} \mathrm{SD}^{29}$ (study primarily based on two previous reviews ${ }^{3031}$ on influenza from 2012 and 2018, respectively) and mathematical modelling studies incorporating the effect of SD. ${ }^{10}{ }^{20-39}$ These models would generally help to 'predict epidemic curve representing the number of infections caused by the virus over time'. ${ }^{40}$

Recently, some SRs and meta-analysis (MA) have been conducted to investigate ethnicity and clinical outcomes. ${ }^{41}$ Chu and colleagues ${ }^{42}$ published an SR including physical distancing to investigate the optimum distance for avoiding person-to-person transmissions, focusing more on face masks and eye protection. Though their study was, perhaps, the first rapidly synthesised review, and identified 172 studies across 16 countries and 6 continents, none of the included studies were randomised controlled trials; therefore, their findings might suffer from both recall and measurement biases. Cochrane further conducted three studies: first, a rapid review in 2020, involving 29 studies on COVID-19 from China, UK, South Korea and Japan. ${ }^{43}$ Second, a rapid qualitative evidence synthesis conduced in 2020 capturing 36 studies from Asia, Africa, Central and North America and Australia examining healthcare workers' adherence and 
enablers or challenges associated with infection control guidelines for respiratory infections. Another study examined 67 studies including randomized controlled trials (RCTs) and observational studies exploring the role of physical interventions for reducing the spread of respiratory viruses and found no evidence regarding screening at entry ports and $\mathrm{SD}{ }^{44}$

Lewnard and $\mathrm{Lo}^{8}$ and Michigan Medicine Projections ${ }^{45}$ reported that combined NPIs using SD, isolation and quarantine, including workplace distancing, appeared effective in reducing COVID-19 compared with no interventions. This approach, however, reported considerable challenges, for example, societal disruption, social isolation/rejection, mental stress and psychological trauma, lack of tests and testing facilities, poor contact tracing and lack of surveillance. No studies examined the combined effects of NPIs in reducing the transmission of COVID-19. This study, therefore, aims to measure the impact of NPIs on reducing COVID-19 transmission.

\section{REVIEW QUESTION}

What has been the impact of NPIs-SD, quarantine and isolation-on reducing transmission of COVID-19?

\section{METHODS AND DESIGNS}

This study will use an SR and MA, which will consider both randomised controlled trials and non-randomised trials (prospective and retrospective observational studies). The Preferred Reporting Items for Systematic Review and Meta-Analysis Protocols statement has been used in the preparation of this protocol (see online supplemental file 1). ${ }^{46}$ Final results will be reported according to the Preferred Reporting Items for Systematic Reviews and Meta-Analyses (PRISMA) statement.

\section{CRITERIA FOR CONSIDERING STUDIES FOR REVIEW Inclusion criteria}

1. Types of participants: this review will consider all studies that involve human subjects of any age-gender, including ethnic (black, Asian and white) and healthcare workers (medical doctors, nurses and allied healthcare professions) groups.

2. Types of intervention: we will include research describing three major NPIs, for example, social distance, isolation and quarantine, focusing only on COVID-19/ SARS-CoV-2.

3. Types of outcome measure. Primary outcomes include: COVID-19; reducing the risk of transmission/ infection of COVID-19; hospitalisation, ICU admissions, COVID-19 related complications and quality of life; and mortality and morbidity. Secondary outcomes include changes in social behaviour, for example, SD by avoiding crowds, restricting movements, isolating ill patients and quarantine of exposed people.
4. Types of studies. No study design filter is added, and there is no limit on our search by language. To measure the impact of NPIs, this review considers all studies evaluating the effectiveness of NPIs relating to reducing the risk of transmission/infection of COVID-19. We include both randomised controlled trials and non-randomised controlled trials, for example, cross-sectional, survey, case-control, randomised controlled trials and observational studies (retrospective or prospective).

(We proposed to collect data from October 2020 to February 2021 for the study).

\section{Exclusion criteria}

1. Articles in narrative reviews, modelling studies, opinion pieces, letters, news, editorials, perspectives, commentaries, conference abstracts and other publications lacking primary data and/or poor methodological details.

2. Studies containing duplicate datasets.

\section{SEARCH STRATEGY TO IDENTIFY RELEVANT STUDIES}

We aim to undertake a systematic search of the following sources: MEDLINE, Embase, Allied \& Complementary Medicine, COVID-19 Research, WHO database on COVID-19, ClinicalTrials. Gov for clinical trials on COVID19, Cochrane Resources on Coronavirus (COVID-19), Oxford COVID-19 Evidence Service and Google Scholar for published and unpublished literatures on COVID-19 including preprint engines such as medRxiv, bioRxiv, Litcovid and SSRN for unpublished studies on COVID-19 will be searched given the lags in publication. The literature search uses the following terms: "social distancing", "quarantine", "isolation", "non-pharmacological interventions" combined with "COVID-19". Primary search terms are non-pharmacological interventions or measures (all synonyms) and COVID-19 (all synonyms) using 'Textword searching'-searching for a word or phrase anywhere in the document, where the document is the citation (article title, journal name and author), not the full text of an article, and 'Thesaurus (MeSH, EMTREE) searching', employing Boolean operators and truncations. The 'Related Articles' feature in PubMed will be consulted. Searches will also be supplemented by reviewing the reference lists ('references of references') of selected articles to find any other relevant papers. From the identified studies in the search, forward and backward citations will also be carried out to find potential studies reporting NPIs and reducing transmission of COVID-19 for the full texts. The literature search strategy was developed by KR in collaboration with departmental subject librarians from authors' universities, who were experienced in SRs, and subsequently refined ensuring its comprehensiveness. While piloting the search strategy, we followed these broad steps:

- Tested out keywords and phrases in a MEDLINE database to see the number of hits returned and assessed the degree of relevance. 
Table 1 Search strategy for MEDLINE

\begin{tabular}{|c|c|}
\hline Concepts & Search terms in each concepts will be modified as needed for use in other databases \\
\hline \multirow[t]{2}{*}{ Concept \#1 } & COVID-19 \\
\hline & 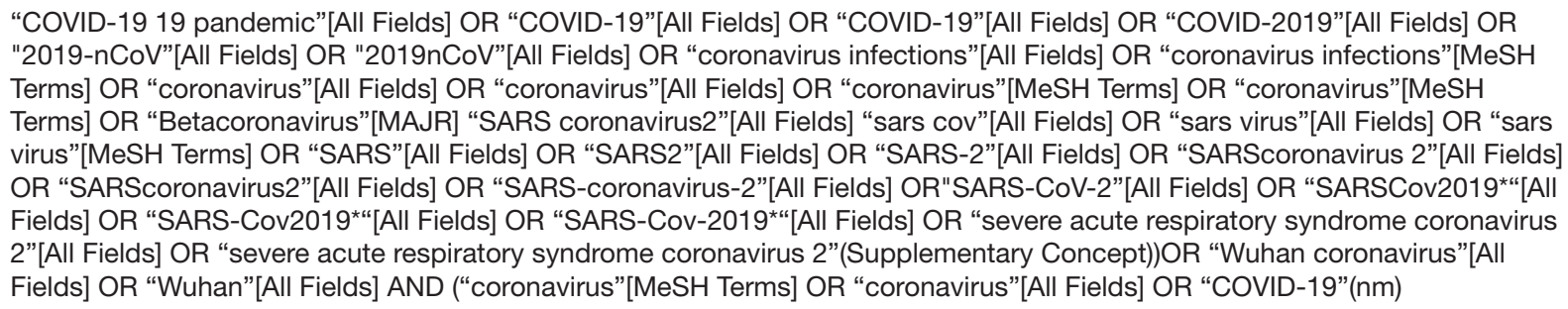 \\
\hline \multirow[t]{2}{*}{ Concept \#2 } & Non-pharmaceutical interventions \\
\hline & $\begin{array}{l}\text { "social distancing"[TIAB] OR "cohorting"[All Fields] OR "community containment"[All Fields] OR "isolation strategy"[All Fields] } \\
\text { OR "isolation"[All Fields] OR "patient isolation"[All Fields] OR "patient isolation"[MeSH Terms] OR "patient isolators"[All Fields] } \\
\text { OR "patient isolators"[MeSH Terms] OR "physical contact"[All Fields] OR "physical distancing"[All Fields] OR "quarantine"[All } \\
\text { Fields] OR "quarantines"[All Fields] OR "quarantine"[MeSH Terms] OR "social distance"[All Fields] OR "quarantines"[All Fields] } \\
\text { OR "quarantined"[All Fields] OR "quarantining"[All Fields] OR "social distance"[MeSH Terms] OR "Social distancing"[All Fields] } \\
\text { OR "Banning"[All Fields] OR "distancing"[All Fields] }\end{array}$ \\
\hline Concept \#3 & 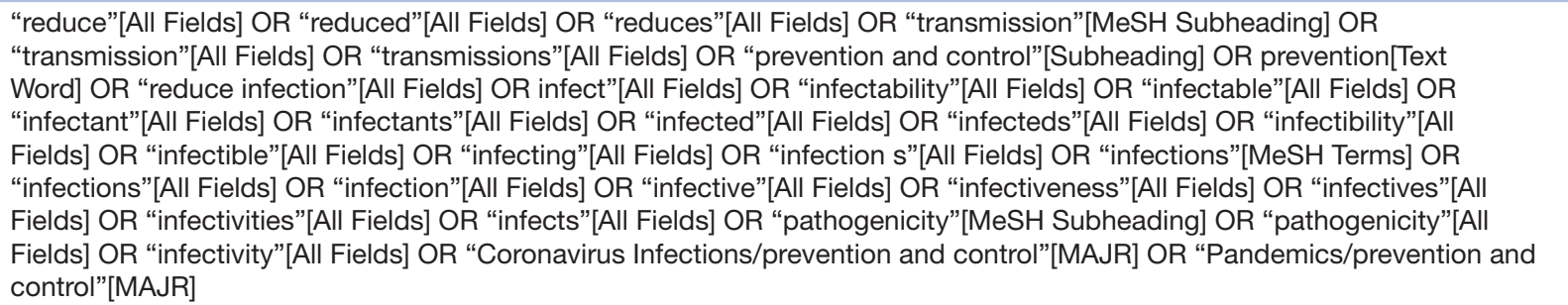 \\
\hline
\end{tabular}

We combined these concepts (using AND), so all concepts are in the same references.

- Reviewed some (eg, five) papers including those marked 'highly cited' on COVID-19/SARS-CoV-2 that meet inclusion/exclusion criteria, where we looked at the terms used in the titles and abstracts for main concepts, for example, NPIs and COVID-19.

- Took notes of keywords supplied by authors and incorporated those into our strategy.

- Experimented with combinations of keywords using 'AND' (limits search) and 'OR' (expands search) operators.

- Looked at subject headings assigned for key papers and used them too.

A broad search strategy has been designed to maximise the level of sensitivity (or comprehensiveness) in searching $^{47}$ and improve both recall ratio (number of relevant references retrieved divided by all of the relevant references) and precision ratio (number of relevant references retrieved divided by the number of references retrieved) (p. 34). ${ }^{48}$ Key terms for one MEDLINE are shown in table 1.

\section{SELECTION OF STUDIES}

The citations identified will be imported into Mendeley Reference Manager (https://www.mendeley.com/). All studies emerging from the databases are screened in two stages: (1) screening of titles and abstracts by two reviewers against minimum inclusion criteria and (2) review of full text. We will use the standard PRISMA flow diagram to provide the study selection process. ${ }^{49}$

\section{QUALITY ASSESSMENT AND RISK OF BIAS}

Quality of the included studies will be assessed using Cochrane Collaboration's tool for assessing risk of bias for randomised controlled trials and the NewcastleOttawa Scale (NOS) for non-randomised studies. ${ }^{50}$ Where possible, we will analyse randomised (according to effectiveness of randomisation method, generation of allocation sequence, allocation concealment, blinding and follow-up) and non-randomised studies (for presence of potential confounders for case-control and cohort studies), and a three-point checklist will be used for controlled before and after studies $^{51}$ separately. In NOS, "a "star system" has been developed in which a study is judged on three broad perspectives: the selection of the study groups; the comparability of the groups; and the ascertainment of either the exposure or outcome of interest for case-control or cohort studies respectively, 50 Some items or questions in these quality assessments, for example, blinded study, are irrelevant to SD studies; we therefore consider removing them. Risk of bias will be examined, as it provides variation, for example, heterogeneity in results of studies included in the study. As Higgins et $a l^{47}$ argue, rigorously conducted studies in the SR would provide more truthful results, and the results from the 
studies of variable validity would give either false negative or false positive conclusions. In this study, the Grading of Recommendations Assessment, Development and Evaluation approach will be used to assess the certainty of the evidence-risk of bias across studies. ${ }^{52}$

Generally, the bias table provides the type of bias (eg, selective reporting of outcomes, random sequence generation, allocation of concealment, blinding of participants, personnel and assessors, incomplete outcome data and other potential threats to validity) in each study. If, for example, most rows are unshaded, then that is considered a low risk of bias, whereas if some rows are either partly shaded or dark (risk of bias either unclear or high), this would provide relatively less confidence in the results. ${ }^{47}$ A narrative synthesis will be conducted for all included studies. Included studies will be assessed by two authors (KR and CML), and the results will inform synthesis and interpretation of the findings. To facilitate comparison of appraisal processes, all reviewers will record the rationale for inclusion or exclusion, and discrepancies will be discussed and resolved by consensus.

\section{ASSESSMENT OF REPORTING BIASES}

Publication bias, often called reporting bias and dissemination bias, is the concern that studies reporting relatively large effects are more likely to be published than studies reporting smaller effects. ${ }^{53}$ Similarly, published studies including multiple outcomes would be more likely to report the outcomes than if they showed statistically significant results. ${ }^{54}$ We will use funnel plot to estimate the publication bias. ${ }^{55}$ If MA had captured all relevant studies, we would expect the funnel plot to be symmetric; that is, we would expect studies to be dispersed equally on either side of the overall effect. ${ }^{53}$ One approach to address publication bias is to follow the trim and fill procedures, that is, assessing asymmetry or symmetry in the funnel plot if more than 10 eligible studies are identified ( $p$. 175) ${ }^{53}$ Trim and fill is a method that allows us to impute these studies; that is, we determine where missing studies are likely to fall, add them to the analysis and then recompute the combined effect. In any MA where the studies are pulled from journals or unpublished data in preprint servers, such as medRxiv, we need to be concerned about the potential impact of publication bias. ${ }^{56-59}$ If the funnel plot is still asymmetric and implies potential bias after including these unpublished data, we use the trim and fill method to quantitatively assess the bias. The trim and fill method serves as a sensitivity analysis. ${ }^{58}$ Specifically, if the smaller studies tend to have larger effects, and if this is actually due to publication bias, this method tells us what the effect size would be in the absence of bias. ${ }^{5759}$

\section{DATA ANALYSIS AND SYNTHESIS}

For quantitative data, where possible, we will measure a risk ratio or $\mathrm{OR}$, absolute risk difference for dichotomous/categorical outcome data, and mean difference or standardised mean difference will be calculated for continuous data, with their $95 \%$ CIs from the data generated by each included study. ${ }^{53}$

If sufficient data are available to make an inference to a universe of comparable studies, results from the comparable groups of studies will be pooled into the statistical random-effects model for MA to measure the effect size of NPIs on reducing transmission of COVID-19 or the strengths of relationships using the software Comprehensive Meta-Analysis V.3. https://www.meta-analysis.com/ pages/new_v3.php?cart=BT2P4569026). The purpose of using a random-effects model in the analysis is 'to incorporate the assumption that the different studies are estimating different, yet related, intervention effects' ${ }^{47}$ To test the heterogeneity of effects in the included studies, we will use Higgins et $a l \mathrm{~s}^{47} \mathrm{I}^{2}$ together with the observed effects to measure the true effects in the analysis. The $\mathrm{I}^{2}$ test for heterogeneity is meant to evaluate whether there is variability across publications.

This will be computed as follows:

$$
\mathrm{I}^{2}=\left(\frac{\mathrm{Q}-\mathrm{df}}{\mathrm{Q}}\right) \times 100 \%
$$

Q-value (Cochran's heterogeneity statistic) is the sum of the squared deviations of all effect sizes from the mean effect size, and $d f$ indicates the degrees of freedom. We report the prediction interval. This speaks directly to the actual utility of the interventions but provides the smallest and largest effect sizes associated with this intervention. ${ }^{60}$ A rough guide, it is interpreted that: $0 \%-40 \%$ might not be important, 30\%-60\% may represent moderate heterogeneity, 50\%-90\% may represent substantial heterogeneity and $75 \%-100 \%$ considerable heterogeneity. ${ }^{47}$ Generally, the importance of observed value of $\mathrm{I}^{2}$ on moderate and substantial heterogeneity depends on the magnitude and direction of effects as well as the strength of heterogeneity. ${ }^{4760}$ Where statistical pooling is not possible, a narrative synthesis is conducted for the included studies. For qualitative data, where meta-synthesis is possible, textual data are pooled using the JBI Qualitative Assessment and Review Instrument and Narrative, Opinion and Text Assessment and Review Instrument. ${ }^{61}$

\section{DATA EXTRACTION AND DATA ITEMS}

Two reviewers independently extract descriptive data and data relevant to the quality of each study using the data extraction form. Data items, that is, source of study, eligibility, reasons for exclusion, methods (study design and duration), participants (number, setting and agegender), intervention and comparator characteristics, results (number of participants, sample size, data for each intervention group and quantitative outcomesmean, SDs and estimate effect), source of funding, ethics approval and study limitation, will be extracted based on the checklist provided by Higgins and Deeks ${ }^{62}$ with appropriate modifications for the review. The data for analysis also include either verbatim quotes directly from participants or the authors' findings. As Rodgers 
and colleagues confirm, this would not only improve the process of transparency by better understanding the sorts of data extracted from which studies, but also recognising the contribution made by each study to the overall synthesis. ${ }^{63}$ In addition, such tables will demonstrate how the individual study area contributes to the reviewers' final conclusion.

\section{DEALING WITH MISSING DATA}

In the case of missing data that might be important to summarise/synthesise study findings, or details of the studies are unclear, we will contact all corresponding authors of included studies to give the opportunity to provide missing data. If authors do not respond, we will record the fact that we tried to contact them,and the number of non-respondents. In such cases, we can either use imputation or risk of bias tools to reduce the likelihood of this being problematic. Generally, it is considered that non-responding authors are equivalent to non-responders to interviews in observational/experimental studies. The impact of this will be reported in the discussion section of the SR.

\section{SUBGROUP ANALYSIS}

We anticipate much variation on the type and nature of NPIs or settings in relation to COVID-19. Based on the scoping search, it is difficult to disentangle the individual effect of each NPI on reducing or preventing COVID-19 transmission, as the role of combined NPIs has been often reported in different literatures; therefore, we do not consider a subgroup analysis to measure which NPIs would be more effective than others. However, some emerging data confirmed cases of COVID-19 and deaths among: (1) different healthcare professionals (medical doctors, nurses and allied healthcare professionals) and (2) socioeconomic groups (black, Asian and white); therefore, we will be doing a subgroup analysis examining the association between NPIs and cases/deaths from COVID-19/ SARS-CoV-2 on those specific groups when applicable. As Higgins et al argue, 'subgroup analyses may be done as a means of investigating heterogeneous results, or to answer specific questions about particular patient groups, types of intervention or type of study' (p. 283). ${ }^{47}$

\section{PATIENT AND PUBLIC INVOLVEMENT}

Patients or the public were not directly involved in the design of this study. As this is a protocol for an SR and no participant recruitment will take place, their involvement on the recruitment and dissemination of findings to participants was not applicable.

\section{ETHICS AND DISSEMINATION}

Formal ethical approval is not required as primary data will not be collected in this study. The final results of this study will be published in an open-access peer-reviewed journal, and abstract will be presented at suitable national/international conferences or workshops.

This SR and MA will report the impact of major NPIs (SD, social isolation and quarantine) on reducing COVID-19 transmission. We will also share important information with public health authorities as well as with the WHO. In addition, we may post the submitted manuscript under review to bioRxiv, medRxiv or other relevant preprint servers.

\section{DISCUSSION}

Impact of NPIs on preventing COVID-19 is a highly charged topic creating much debate among politicians, economists, and medical and public health professions. Given the rapidly growing field, it is imperative to generate a substantial conclusion regarding the prevention, control and management of COVID-19 in public health practice. The proposed SR will therefore measure the impact of NPIs on reducing transmission of COVID-19. As such, significant outcomes from this review will guide patients and clinicians in their treatment arrangements given that there is no vaccine or treatment available at the time of writing. Furthermore, these significant findings will be vital to assist policymakers and researchers in synthesising a large and complex literature. Similarly, this review will provide a basis for developing the best methods and approaches for developing objective measures and interventions to establish the link between different factors and NPIs and reducing transmission of COVID-19 effectively, efficiently and equitably. It is equally important that the 'structure and capacity of our depleted healthcare system are now largely driving the response to this epidemic ${ }^{\text {,64 }}$ and most likely it will continue to do so until services that support local communicable disease control are rebuilt and reintegrated ${ }^{65}$ It is, therefore, important to make appropriate efforts now that would address COVID-19, through strengthening the primary healthcare system, to reduce the chances of future pandemics.

\section{Twitter Cho Mar Lwin@Cho Mar Lwin}

Acknowledgements We would like to thank the reviewers of the manuscript for their constructive feedback. We would like to thank Michael Borenstein, Lifeng Lin, Paul Levay, Jesus N Sarol Jr and David Hewson for their inputs in the protocol. We would also like to thank our subject librarians especially, David Abdy, for his help with finalising the search strategy.

Contributors KR conceived and designed the research with the advice from CML; $\mathrm{KR}$ wrote the first draft; KR and CML reviewed and contributed to drafting, revising and finalising the manuscript. Both authors have reviewed and approved the final version of the manuscript and have given their permission for publication.

Funding The authors have not declared a specific grant for this research from any funding agency in the public, commercial or not-for-profit sectors.

Competing interests None declared.

Patient consent for publication Not required.

Provenance and peer review Not commissioned; externally peer reviewed.

Supplemental material This content has been supplied by the author(s). It has not been vetted by BMJ Publishing Group Limited (BMJ) and may not have been peer-reviewed. Any opinions or recommendations discussed are solely those 
of the author(s) and are not endorsed by BMJ. BMJ disclaims all liability and responsibility arising from any reliance placed on the content. Where the content includes any translated material, BMJ does not warrant the accuracy and reliability of the translations (including but not limited to local regulations, clinical guidelines, terminology, drug names and drug dosages), and is not responsible for any error and/or omissions arising from translation and adaptation or otherwise.

Open access This is an open access article distributed in accordance with the Creative Commons Attribution Non Commercial (CC BY-NC 4.0) license, which permits others to distribute, remix, adapt, build upon this work non-commercially, and license their derivative works on different terms, provided the original work is properly cited, appropriate credit is given, any changes made indicated, and the use is non-commercial. See: http://creativecommons.org/licenses/by-nc/4.0/.

\section{ORCID iD}

Krishna Regmi http://orcid.org/0000-0002-1408-1978

\section{REFERENCES}

1 World Health Organisation. WHO Director-General's opening remarks at the media briefing on COVID-19, 2020. Available: https://www. who.int/dg/speeches/detail/who-director-general-s-openingremarks-at-the-media-briefing-on-covid-19-11-march-2020

2 World Heath Organisation. Coronavirus disease (COVID-19) pandemic, 2020. Available: https://www.who.int/emergencies/ diseases/novel-coronavirus-2019

3 Razaq A, Harrison D, Karunanithi S, et al. BAME COVID-19 deathswhat do we know? Rapid data \& evidence review: "hidden in plain sight.", 2020. Available: https://www.cebm.net/wp-content/uploads/ 2020/05/BAME-COVID-Rapid-Data-Evidence-Review-Final-Hiddenin-Plain-Sight-compressed.pdf

4 Cook T, Kursumovic E, Lennane S. Exclusive: deaths of NHS staff from covid-19 analysed, 2020. Available: https://www.hsj.co.uk/ exclusive-deaths-of-nhs-staff-from-covid-19-analysed/7027471. article

5 Public Health England. Beyond the data: understanding the impact of COVID-19 on BamE communities, 2020. Available: https://assets. publishing.service.gov.uk/government/uploads/system/uploads/ attachment_data/file/892376/COVID_stakeholder_engagement_ synthesis_beyond the_data.pdf

6 Public Health England. Disparities in the risk and outcomes from COVID-19, 2020. Available: https://assets.publishing.service.gov.uk/ government/uploads/system/uploads/attachment_data/file/889195/ disparities_review.pdf

7 van Elsland SL, O'Hare R. Coronavirus pandemic could have caused 40 million deaths if left unchecked, 2020. Available: https://www. imperial.ac.uk/news/196496/coronavirus-pandemic-could-havecaused-40/

8 Lewnard JA, Lo NC. Scientific and ethical basis for social-distancing interventions against COVID-19. Lancet Infect Dis 2020;20:631-3.

9 Feuer W. Worldwide coronavirus cases top 200,000, doubling in two weeks, 2020. Available: https://www.cnbc.com/2020/03/18/ worldwide-coronavirus-cases-top-200000-for-the-first-time.htm

10 Flaxman S, Mishra S, Gandy A, et al. Estimating the effects of non-pharmaceutical interventions on COVID-19 in Europe. Nature 2020;584:257-61.

11 World Health Organization. Overview of public health and socia measures in the context of COVID-19: interim guidance, 2020. Available: https://apps.who.int/iris/handle/10665/332115

12 Akiyama MJ, Spaulding AC, Rich JD. Flattening the Curve for Incarcerated Populations - Covid-19 in Jails and Prisons. N Engl J Med 2020;382:2075-7.

13 Chowdhury A, Kabir K, Tanimoto J, et al. How quarantine and social distancing policy can suppress the outbreak of novel coronavirus in developing or under poverty level countries: a mathematical and statistical analysis, 2020

14 Hick JL, Biddinger PD. Novel coronavirus and old lessons - preparing the health system for the pandemic. N Engl J Med 2020;382:e55.

15 Ahmed F, Zviedrite N, Uzicanin A. Effectiveness of workplace social distancing measures in reducing influenza transmission: a systematic review. BMC Public Health 2018;18:518.

16 Mal PR, Suneel P, Shomeeta P. Social distancing: a nonpharmacological intervention for COVID-19. J Pak Med Assoc 2020;70:S21-4.

17 Kar SK, Arafat SMY, Sharma P, et al. COVID-19 pandemic and addiction: current problems and future concerns. Asian J Psychiatr 2020;51:102064.

18 Harris M, Ghebreyesus T, Ryan M, et al. Covid-19, 2020. Available https://web.archive.org/web/20200325084602/https://www.who. int/docs/default-source/coronaviruse/transcripts/who-audioemergencies-coronavirus-press-conference-full-20mar2020.pdf? sfvrsn=1eafbff_0

19 Johnson CY. SLFA. social distancing could buy U.S. valuable time against coronavirus, 2020. Available: https://www.washingtonpost. com/health/2020/03/10/social-distancing-coronavirus/

20 Rocklöv J, Sjödin H, Wilder-Smith A. COVID-19 outbreak on the diamond Princess cruise SHIP: estimating the epidemic potential and effectiveness of public health countermeasures. J Travel Med 2020;27. doi:10.1093/jtm/taaa030. [Epub ahead of print: 18 May 2020].

21 Sjödin H, Wilder-Smith A, Osman S, et al. Only strict quarantine measures can curb the coronavirus disease (COVID-19) outbreak in Italy, 2020. Euro Surveill 2020;25:2000280.

22 World Health Organisation. Coronavirus disease (COVID-19) advice for the public, 2020. Available: https://www.who.int/emergencies/ diseases/novel-coronavirus-2019/advice-for-public

23 Park M, Cook AR, Lim JT, et al. A systematic review of covid-19 epidemiology based on current evidence. J Clin Med 2020;9:967.

24 Harapan $\mathrm{H}$, Itoh N, Yufika A, et al. The COVID-19 pandemic calls for spatial distancing and social closeness: not for social distancing! $J$ Infect Public Health 2020;13:667-73.

25 Lin C, Ye R, Xia YL. A meta-analysis to evaluate the effectiveness of real-time PCR for diagnosing novel coronavirus infections. Genet Mol Res 2015;14:15634-41.

26 Viner RM, Russell SJ, Croker H, et al. School closure and management practices during coronavirus outbreaks including COVID-19: a rapid systematic review. Lancet Child Adolesc Heal 2020;4:397-404

27 Brooks SK, Webster RK, Smith LE, et al. The psychological impact of quarantine and how to reduce it: rapid review of the evidence. Lancet 2020;395:912-20.

28 Webster RK, Brooks SK, Smith LE, et al. How to improve adherence with quarantine: rapid review of the evidence. medRxiv 2020.

29 Mahtani KR, Heneghan C, Aronson JK. What is the evidence for social distancing during global pandemics? a rapid summary of current knowledge, 2020. Available: https://www.phc.ox.ac.uk/ files/covid-19-evidence-service/what-is-the-evidence-for-socialdistancing-during-global-pandemics-final-1.pdf/view

30 Rashid H, Ridda I, King C, et al. Evidence compendium and advice on social distancing and other related measures for response to an influenza pandemic. Paediatr Respir Rev 2015;16:119-26.

31 Fong MW, Gao H, Wong JY, et al. Nonpharmaceutical measures for pandemic influenza in nonhealthcare settings-social distancing measures. Emerg Infect Dis 2020;26:976-84.

32 Ferguson N, Laydon D, Nedjati-Gilani G, et al. Report 9 - Impact of non-pharmaceutical interventions (NPIs) to reduce COVID-19 mortality and healthcare demand, 2020. Available: https://www. imperial.ac.uk/mrc-global-infectious-disease-analysis/covid-19/ report-9-impact-of-npis-on-covid-19/

33 Lai S, Ruktanonchai NW, Zhou L, et al. Effect of non-pharmaceutical interventions to contain COVID-19 in China. Nature 2020;585:410-3.

34 Bayham J, Fenichel EP. Impact of school closures for COVID-19 on the US health-care workforce and net mortality: a modelling study. Lancet Public Heal 2020;5:e271-8.

35 Hellewell J, Abbott S, Gimma A, et al. Feasibility of controlling COVID-19 outbreaks by isolation of cases and contacts. Lancet Glob Heal 2020;8:e488-96.

36 Prem K, Liu Y, Russell TW, et al. The effect of control strategies to reduce social mixing on outcomes of the COVID-19 epidemic in Wuhan, China: a modelling study. Lancet Public Heal 2020;5:e261-70.

37 Koo JR, Cook AR, Park M, et al. Interventions to mitigate early spread of SARS-CoV-2 in Singapore: a modelling study. Lancet Infect Dis 2020;20:678-88.

38 Tang B, Xia F, Tang S, et al. The effectiveness of quarantine and isolation determine the trend of the COVID-19 epidemics in the final phase of the current outbreak in China. Int $\mathrm{J}$ Infect Dis 2020;95:288-93.

39 Tang B, Wang X, Li Q, et al. Estimation of the transmission risk of the 2019-ncov and its implication for public health interventions. J Clin Med 2020;9:462.

40 Panovska-Griffiths J. Can mathematical modelling solve the current Covid-19 crisis? BMC Public Health 2020;20:551.

41 Pan D, Sze S, Minhas JS, et al. The impact of ethnicity on clinical outcomes in COVID-19: a systematic review. EClinicalMedicine 2020;23:100404.

42 Chu DK, Akl EA, Duda S, et al. Physical distancing, face masks, and eye protection to prevent person-to-person transmission of SARS-CoV-2 and COVID-19: a systematic review and meta-analysis. Lancet 2020;395:1973-87. 
43 Nussbaumer-Streit B, Mayr V, Dobrescu Al, et al. Quarantine alone or in combination with other public health measures to control COVID-19: a rapid review. Cochrane Database Syst Rev 2020;4:CD013574.

44 Jefferson T, Del Mar CB, Dooley L, et al. Physical interventions to interrupt or reduce the spread of respiratory viruses. Cochrane database Syst Rev 2011;141.

45 University of Michigan. Michigan medicine projections show aggressive social distancing will dramatically reduce the peak number of hospitalized COVID-19 patients, 2020. Available: https:// www.uofmhealth.org/news/archive/202003/michigan-medicineprojections-show-aggressive-social

46 Shamseer L, Moher D, Clarke M, et al. Preferred reporting items for systematic review and meta-analysis protocols (PRISMA-P) 2015: elaboration and explanation. BMJ 2015;349:g7647

47 Higgins JPT, Thomas J, Chandler J, et al. Cochrane Handbook for systematic reviews of interventions. Chichester, West Sussex: John Wiley \& Sons, 2019.

48 Katcher BS. Medline: a guide to effective searching in pubmed \& other interfaces. San Francisco, CA: Ashbury Press, 2006.

49 Moher D, Liberati A, Tetzlaff J, et al. Preferred reporting items for systematic reviews and meta-analyses: the PRISMA statement. BMJ 2009;339:b2535-336.

50 Wells G, Shea B, O'Connell D, et al. The Newcastle-Ottawa scale (NOS) for assessing the quality of nonrandomised studies in metaanalyses, 2019. Available: http://www.ohri.ca/programs/clinical_ epidemiology/oxford.asp

51 Khan SK, ter Riet G, Popay J, et al. Stage II Conducting the review: phase 5: study quality assessment. In: Khan SK, ter Riet G, Glanville J, et al, eds. Undertaking systematic reviews of research on effectiveness. CRD's guidance for carrying out or commissioning reviews. 2nd ed. York: University of York, 2000.

52 Guyatt GH, Oxman AD, Vist GE, et al. Grade: an emerging consensus on rating quality of evidence and strength of recommendations. BMJ 2008;336:924-6.
53 Borenstein M. Common mistakes in meta-analysis and how to avoid them. USA: Biostat, Inc, 2019.

54 Sterne J, Egger M, Moher D. Addressing reporting biases. Wiley, 2008.

55 Egger M, Davey Smith G, Schneider M, et al. Bias in meta-analysis detected by a simple, graphical test. BMJ 1997;315:629-34.

56 Peters JL, Sutton AJ, Jones DR, et al. Assessing publication bias in meta-analyses in the presence of between-study heterogeneity. J $R$ Stat Soc Ser A Stat Soc 2010;173:575-91.

57 Jackson D. The implications of publication bias for meta-analysis' other parameter. Stat Med 2006;25:2911-21.

58 Peters JL, Sutton AJ, Jones DR, et al. Performance of the TRIM and fill method in the presence of publication bias and between-study heterogeneity. Stat Med 2007;26:4544-62.

59 Terrin N, Schmid CH, Lau J, et al. Adjusting for publication bias in the presence of heterogeneity. Stat Med 2003;22:2113-26.

60 Borenstein M, Hedges L, Higgins J, et al. Introduction to metaanalysis. Chichester, West Sussex: John Wiley \& Sons, 2009.

61 Joanna Briggs Institute. The Joanna Briggs Institute Reviewers' manual 2014: methodology for JBI mixed methods systematic reviews. Adelaide, Australia: Joanna Briggs Institute, 2014.

62 Higgins J, Deeks J. Chapter 7: Selecting Studies and Collecting Data. In: Higgins J, Greens S, eds. Cochrane Handbook for systematic reviews of interventions. Chichester, West Sussex: John Wiley \& Sons, 2008.

63 Rodgers M, Sowden A, Petticrew M, et al. Testing methodological guidance on the conduct of narrative synthesis in systematic reviews. Evaluation 2009;15:49-73.

64 Pollock AM, Roderick P, Cheng KK, et al. Covid-19: why is the UK government ignoring who's advice? BMJ 2020;368:m1284.

65 Regmi K, Gilbert R, Thunhurst C. How can health systems be strengthened to control and prevent an Ebola outbreak? A narrative review. Infect Ecol Epidemiol 2015;5:28877. 